\title{
A NOVEL ROLE OF PLASMA MEMBRANE CALCIUM ATPASE 4 AS A NEGATIVE- REGULATOR OF VEGF-INDUCED ANGIOGENESIS
}

RR Baggott ${ }^{1}$, A Alfranca ${ }^{2,3}$, MD López-Maderuelo² ${ }^{2}$ TMA Mohamed ${ }^{4,5}$, A Escolano ${ }^{2}$, J Oller ${ }^{2}$, BC Ornés², FB Rowther ${ }^{1}$, D Oceandy ${ }^{4}$, S Kurusamy ${ }^{1}$, J Brown ${ }^{6}$, EJ Cartwright ${ }^{4}$, W Wang ${ }^{1}$, P Gómez-Del Arco ${ }^{2,7}$, S Martínez-Martínez ${ }^{2}$, L Neyses $^{4,8}$, JM Redondo ${ }^{2}$, AL Armesilla $^{1}$

${ }^{I}$ Research Institute in Healthcare Science, School of Pharmacy, Faculty of Science and Engineering, University of Wolverhampton, Wolverhampton, UK; ${ }^{2}$ Department of Vascular Biology and Inflammation, Centro Nacional de Investigaciones Cardiovasculares CNIC, Madrid, Spain; ${ }^{3}$ Human Genetics Department Institute for Rare Diseases Research, Carlos III Health Institute, Madrid, Spain; ${ }^{4}$ Cardiovascular Research Group, University of Manchester, Manchester, UK; ${ }^{5}$ Department of Biochemistry, Faculty of Pharmacy, Zagazig University, Zagazig, Egypt; ${ }^{6}$ Aston Research Centre for Healthy Ageing, Aston University, Birmingham, UK; ${ }^{7}$ Department of Molecular Biology, Universidad Autónoma de Madrid; ${ }^{8}$ University of Luxembourg

Current anti-angiogenic treatments involve the attenuation of signalling via the pro-angiogenic vascular endothelial growth factor/receptor (VEGF/VEGFR) axis. Stimulation of angiogenesis by VEGF requires the activation of the calcineurin/nuclear factor of activated T-cells (NFAT) signal transduction pathway which is inhibited by Plasma Membrane Calcium ATPase 4 (PMCA4), an endogenous calcium extrusion pump. However, PMCA4s role in calcineurin/NFAT-dependent angiogenesis is unknown. Using "gain of function" studies, we show here that adenoviral overexpression of PMCA4 in human umbilical vein endothelial cells (HUVEC) inhibited NFAT activity, decreased the expression of NFAT-dependent pro-angiogenic proteins (regulator of calcineurin 1.4 (RCAN1.4) and cyclooxygenase-2) and diminished in vitro cell migration and tube formation in response to VEGF-stimulation. Furthermore, in vivo blood vessel formation was attenuated in a matrigel plug assay by ectopic expression of PMCA4. Conversely, "loss of function" experiments by si-RNA-mediated knockdown of PMCA4 in HUVEC or isolation of mouse lung endothelial cells from PMCA4-/- mice showed increased VEGF-induced NFAT activity, RCAN1.4 expression, in vitro endothelial cell migration, tube formation and in vivo blood vessel formation. Additionally, in an in vivo pathological angiogenesis model of limb ischemia, the reperfusion of the ischemic limb of PMCA4-/- mice was augmented compared to wild-type. Disruption of the interaction between endogenous PMCA4 and calcineurin by adenoviral overexpression of the region of PMCA4 that interacts with calcineurin (residues 428-651) increased NFAT activity, RCAN1.4 protein expression and in vitro tube formation. These results identify PMCA4 as an inhibitor of VEGF-induced angiogenesis, highlighting its potential as a new therapeutic target for anti-angiogenic treatments. 